\title{
Generalized quasi-Banach sequence spaces and measures of noncompactness
}

\author{
EDUARDO B. SILVA ${ }^{1}$, DIOCESAR L. FERNANDEZ ${ }^{2}$ and LUDMILA NIKOLOVA ${ }^{3}$ \\ ${ }^{1}$ Universidade Estadual de Maringá, DMA, Av. Colombo, 5790, 87020-900 Maringá, PR, Brasil \\ ${ }^{2}$ Universidade Estadual de Campinas, IMECC, Caixa Postal 6065, 13083-859 Campinas, SP, Brasil \\ ${ }^{3}$ Faculty of Mathematics and Informatics, Sofia University, Blvd. James Bourchier, 5, 1164 Sofia, Bulgaria
}

Manuscript received on November 28, 2011; accepted for publication on July 17, 2012

\begin{abstract}
Given $0<s \leq 1$ and $\psi$ an $s$-convex function, $s-\psi$-sequence spaces are introduced. Several quasi-Banach sequence spaces are thus characterized as a particular case of $s-\psi$-spaces. For these spaces, new measures of noncompactness are also defined, related to the Hausdorff measure of noncompactness. As an application, compact sets in $s-\psi$-interpolation spaces of a quasi-Banach couple are studied.
\end{abstract}

Key words: quasi-Banach spaces, quasi-convex functions, measures of noncompactness, interpolation theory.

\section{INTRODUCTION}

Lately many researchers have been interested about diverse issues related to quasi-Banach spaces. These spaces arise in a natural way as a generalization of Banach spaces, where the triangular inequality of the norm is changed by a weaker condition. From a geometrical point of view, the convex unitary ball of the Banach space case is replaced in the quasi-Banach case by a nonconvex unitary ball. Besides the classical works by (Aoki 1942), (Rolewicz 1957, 1985) and (Kalton et al. 1985), the study of geometrical aspects is one of the main issues for these spaces, with several results obtained recently, as it may be seen in the works by Albiac and Kalton (2009), Albiac and Leranóz (2010a, b) and Mastylo and Mleczko (2010). Results on quasi-Banach spaces have been applied in related subjects, for example, to obtain important characterizations on $H^{p}$ spaces, for $0<p \leq 1$, as it may be seen in (Bownik 2005), (Bownik et al. 2010) and (Gomez and Silva 2011).

Another example of quasi-Banach spaces cames from the interpolation theory. In the case of abstract Banach spaces and operators, this theory began with the classical papers by Lions and Peetre (1964), and Calderón (1964), constituting a very active research field.

The study of geometrical aspects of interpolated spaces is one of the main issues. Besides the normed case, the research about the behavior of quasi-Banach spaces under interpolation methods begun with papers by Krée (1967), Holmsted (1970), Peetre (1970) and Sagher (1972). More recently, the subject has attracted a lot of attention, since several properties and issues from the normed case like interpolation of bilinear

AMS Classification: Primary 46A45, 46B70, 47H08, 46E30.

Correspondence to: Eduardo Brandani da Silva

E-mail: ebsilva@uem.br 
operators, geometric aspects, maximal and minimal functors and compactness, have been generalized to the quasi-Banach case. See, for instance, the works of Bergh and Cobos (2000), Cobos et al. (2007), Grafakos and Mastylo (2006), Ghorbani and Modarres (2007), Molina (2009) and Cobos and Persson (1998).

Sequence Banach spaces are also another very active research subject, deeply connected with several definitions, characterizations and properties in functional analysis. In a very interesting paper, Mitani and Saito (2007) introduced a class of sequence spaces, called the $\ell_{\psi}$ sequence spaces, which presents in a unified form, norm and geometric properties of several well-known Banach sequence spaces. Recent papers by Nikolova and Zachariades (2009) and Zachariades (2011) presents an interpolation theory of couples of Banach spaces modeled on $\ell \psi$ spaces and characterizes several geometric properties. These spaces possess good generality and are very workable for a unified application, but the constructions of Mitani and Saito (2007), Nikolova and Zachariades (2009) and Zachariades (2011) are not directly generalized to quasiBanach sequence spaces.

The notion of measure of noncompactness was introduced by K. Kuratowski. The Kuratowski measure, as well as its variant, called by some authors the Haudorff measure, has a very important role in functional analysis. It is applied to the theories of differential and integral equations as well as to the operator theory. The relation between measures of noncompactness and interpolation theory of linear and non-linear operators is a very active research topic. See, for example Banás and Goebel (1980) and Fernandez and Silva (2010) and the references therein.

In current work, given $0<s \leq 1$ and an s-convex function $\psi$, the $s-\psi$-sequence spaces are introduced. A necessary condition is given which guarantees the existence of these spaces and some properties are proved, including that they are quasi-Banach spaces. This allow us to characterize some quasi-Banach sequence spaces as a particular case of $s-\psi$-spaces.

New measures of noncompactness related to Hausdorff measure of noncompactness are also introduced to obtain a quantitative version of a classical result by Phillips (1940, Thm. 3.7) [see also Dunford and Schwartz (1967 Lemma IV.5.4, p. 259)] and Brooks and Dinculeanu (1979, Thm. 1). These quantitative results for the quasi-Banach case seems to be new in the literature.

Interpolation spaces on $s^{-} \psi$-sequence spaces are also defined. Compact sets in this interpolation spaces are investigated and a characterization of them is obtained.

\section{S-CONVEX FUNCTIONS AND $\psi$-SEQUENCE SPACES}

Let us set

$$
c_{00}=\left\{z=\left(z_{n}\right)_{n \in} \mathbb{N} \in \mathbb{C}^{\mathbb{N}}: z_{n} \neq 0 \text { for finite number of } n \in \mathbb{N}\right\},
$$

and let $\left(e_{n}\right)_{n \in \mathbb{N}}$ be the usual basis of $c_{00}$. Denote $|z|=\left(\left|z_{n}\right|\right)_{n \in N}$.

DEFINITION 1. Given $0<s \leq 1$, an s-norm on $c_{00}$ is a functional $\|\|:. c_{00} \rightarrow \mathbb{R}_{+}$.satisfying

(a) $\|x\| \geq 0$ for all $x \in c_{00}$ and $\|x\|=0$ if, and only if $x=0$.

(b) $\|\lambda x\|=|\lambda|\|x\|$ for all $\lambda \in \mathbb{R}$ and $x \in c_{00}$.

(c) $\|x+y\|^{s} \leq\|x\|^{s}+\|y\|^{s}$ for all $x, y \in c_{00}$.

An s-norm on $c_{00}$ is called absolute if $\|z\|=\||z|\|$ for every $z \in c_{00}$ and it is called normalized if $\left\|e_{i}\right\|=1$, for all $i \in \mathbb{N}$. 
DEFINITION 2. Given $x=\left(x_{i}\right)_{i \in \mathbb{N}}, \mathrm{y}=\left(y_{i}\right)_{i \in \mathbb{N}} \in c_{00}$, then $|x| \leq|y|$ if, and only if $\left|x_{i}\right| \leq\left|y_{i}\right|, \forall_{i} \in \mathbb{N}$. An absolute norm on $c_{00}$ is monotone if $|x| \leq|y|$ implies $\|x\| \leq\|y\|$.

DEFINITION 3. Given $0<s \leq 1$, let $\mathcal{A C}_{s, \infty}$ be the set of all absolute and monotone s-norms on $c_{00}$ such that, for all $e_{i}$, there are positive constants $c_{1}$ and $c_{2}$ satisfying

$$
0<c_{1} \leq\left\|e_{i}\right\| \leq c_{2} .
$$

Let $\mathcal{A N}_{s, \infty}$ be the set of all absolute, normalized and monotone $s$-norms on $c_{00}$. We denote by $\Delta_{\infty}$ the set

$$
\Delta_{\infty}=\left\{p=\left(p_{n}\right)_{n \in \mathbb{N}} \in c_{00}: \sum_{n \in \mathbb{N}} p_{n}=1, p_{n} \geq 0\right\} .
$$

LEMMA 1. For all $\|.\| \in \mathcal{A C}_{\mathrm{s}, \infty}$ one has

$$
c_{1}\|\cdot\|_{\infty} \leq\|\cdot\| \leq c_{2}\|\cdot\|_{s},
$$

where $\|\cdot\|_{\mathrm{s}}$ and $\|\cdot\|_{\infty}$ are the norms on $\ell_{\mathrm{s}}$ and $\ell_{\infty}$, respectively.

PROOF. Given $x=\left(x_{n}\right)_{n \in \mathbb{N}} \in c_{00}$, let us suppose $x_{n}=0$ for $n>m$ and $\|x\|_{\infty}=\left|x_{1}\right|$. Then,

$$
\begin{aligned}
\|x\|_{\infty} & =\left|x_{1}\right|=\left|x_{1}\right| \frac{\left\|e_{1}\right\|}{\left\|e_{1}\right\|}=\frac{1}{\left\|e_{1}\right\|}\left\|\left(\left|x_{1}\right|, 0,0, \ldots\right)\right\| \\
& \leq \frac{1}{c_{1}}\left\|\left(\left|x_{1}\right|, 0,0, \cdots\right)\right\| \\
& \leq \frac{1}{c_{1}}\left\|\left(\left|x_{1}\right|,\left|x_{2}\right|,\left|x_{3}\right|, \cdots\right)\right\| \\
& =\frac{1}{c_{1}}\left\|\left(\left|x_{1}\right|,\left|x_{2}\right|,\left|x_{3}\right|, \cdots\right)\right\|=\frac{\|x\|}{c_{1}} .
\end{aligned}
$$

Now,

$$
\begin{aligned}
\|\left.\left(x_{n}\right)_{n \in \mathbb{N}}\right|^{s} & =\|\left|x_{1}\right| e_{1}+\left.\left(0,\left|x_{2}\right|,\left|x_{3}\right|, \cdots\right)\right|^{s} \\
& \leq\left\|\left|x_{1}\right| e_{1}||^{s}+\left(0,\left|x_{2}\right|,\left|x_{3}\right|, \cdots\right)\right\|^{s} \\
& \leq\left|x_{1}\right|^{s}\left\|\left.e_{1}\right|^{s}+\left(0,\left|x_{2}\right|,\left|x_{3}\right|, \cdots\right)\right\|^{s} \\
& \leq\left|x_{1}\right|^{s} c_{2}^{s}+||\left|x_{2}\right| e_{2}||+\left(0,0,\left|x_{3}\right|, \cdots\right) \|^{s} \\
& \leq\left|x_{1}\right|^{s} c_{2}^{s}+\left|x_{2}\right|^{s}\left\|e_{2}||^{s}+\right\|\left(0,0,\left|x_{3}\right|, \cdots\right) \|^{s} \\
& \leq\left|x_{1}\right|^{s} c_{2}^{s}+\left|x_{2}\right|^{s}\left\|c_{2}||^{s}+\left(0,0,\left|x_{3}\right|, \cdots\right)\right\|^{s} \\
& \leq \cdots \\
& \leq c_{2}^{s}\left(\left|x_{1}\right|^{s}+\left|x_{2}\right|^{s}+\cdots+\left|x_{m}\right|^{s}\right) \\
& =c_{2}^{s}\|x\|_{\ell_{s}}^{s} .
\end{aligned}
$$

DEFINITION 4. Given $0<s \leq 1$, a linear space V and a function $f: V \rightarrow \mathbb{R}$, then $f$ is said to be s-convex in the second sense if inequality

$$
f(\alpha u+\beta v) \leq \alpha^{s} f(u)+\beta^{s} f(v)
$$


holds for all $u, v \in V$ and $\alpha, \beta \geq 0$ with $\alpha+\beta=1$. The set of all these functions is denoted by $K_{s}^{2}(V)$.

If $s=1$ the definition means just convexity. Another useful result is

PROPOSITION 1. If $f \in K_{2}^{s}(V)$ and $0<s<1$, then $f$ is non-negative on $V$.

Proof of this fact and several another results about $s$-convex functions appear in (Hudzik and Maligranda 1994).

DEFINITION 5. Given $0<s \leq 1, \Psi_{s, \infty}$ will be the set of all s-convex continuous functions $\psi$ (in the second sense) on $\Delta_{\infty}$, for which $\psi\left(e_{n}\right)=1$ and condition (A) is fulfilled, namely

$$
\begin{gathered}
\lambda^{\mathrm{s}} \psi\left(t_{1}, \ldots, t_{i-1}, t_{i}, t_{i+1}, \ldots, t_{n}, \ldots\right) \geq \\
\psi\left(\lambda t_{1}, \ldots, \lambda t_{i-1},(1-\lambda)+\lambda t_{i}, \lambda t_{i+1}, \ldots, \lambda t_{n}, \ldots\right),
\end{gathered}
$$

for all $\lambda$, where $1<\lambda \leq \frac{1}{1-t_{i}}, i \in \mathbb{N}$ and $t=\left(\mathrm{t}_{i}\right)_{i \in \mathbb{N}} \in \Delta_{\infty}$ with $t_{i} \neq 1$.

DEFINITION 6. Given $\psi \in \Psi_{s, \infty}$, the function $\psi_{n}: \mathbb{R}^{n} \rightarrow \mathbb{R}_{+}$is defined by

$$
\psi_{n}\left(x_{1}, \ldots, x_{n}\right)=\psi\left(x_{1}, \ldots, x_{n}, 0, \ldots, 0\right),\left(x_{i}\right)_{i \in \mathbb{N}} .
$$

THEOREM 1. (i). For every $\|.\| \in \mathcal{A N}_{s, \infty}$, we define $\psi: \Delta_{\infty} \rightarrow \mathbb{R}+$ by

$$
\psi(p)=\|p\|^{s} .
$$

Then $\psi \in \Psi_{s, \infty}$.

(ii)For any $\psi \in \Psi_{s, \infty}$ and $x \in c_{00}$ we define

$$
\left\|\left(x_{i}\right)_{i \in \mathbb{N}}\right\|_{\psi}^{S}= \begin{cases}\left(\sum_{i=1}^{\infty}\left|x_{i}\right|\right)^{s} \psi\left(\frac{\left|x_{1}\right|}{\sum_{i=1}^{\infty}\left|x_{i}\right|}, \ldots, \frac{\left|x_{1}\right|}{\sum_{i=1}^{\infty}\left|x_{i}\right|}, \ldots\right), & \text { if }\left(x_{n}\right)_{n \in \mathbb{N}} \neq 0 \\ 0, & \text { if }\left(x_{n}\right)_{n \in \mathbb{N}}=0 .\end{cases}
$$

Then, $\|.\| \psi \in \mathcal{A N}_{s, \infty}$ and satisfies (2).

Therefore, $\mathcal{A} \mathcal{N}_{s, \infty}$ and $\Psi_{s, \infty}$ are in a one-to-one correspondence under the equation 2.9(2).

PROOF. (i) Given $t=\left(t_{i}\right)_{i \in \mathbb{N}} \in \Delta_{\infty}$, with $t_{i} \neq 1$ and $\lambda$ such that $1<\lambda \leq \frac{1}{1-t_{i}}$, for all $i \in \mathbb{N}$, then $\lambda \leq \frac{1}{1-t_{i}}$ implies $\lambda t_{i} \geq(1-\lambda)+\lambda t_{i} \geq 0$. Since $\|.\| \in \mathcal{A N}_{s, \infty}$, the norm is monotone and one has

$$
\left\|\left\{\lambda t_{\mathrm{i}}\right\}\right\|^{s} \geq\left\|\lambda t_{1}, \ldots, \lambda t_{i-1},(1-\lambda)+\lambda t_{i}, \lambda t_{i}+1, \ldots, \lambda t_{n}\right\|^{s},
$$

and so the condition (A) holds.

(ii) From the definition of $\|.\|_{\psi}$ the properties (a) and (b) of Definition 2.1 are verified. Now, let $x \in c_{00}$. There exists some $n \in \mathbb{N}$ with $x_{i}=0$ for all $i>n$. Then $\left\|\left(x_{i}\right)_{i=1}^{\infty}\right\|_{\psi}^{s}={ }^{n}\left\|\left(x_{i}\right)_{i=1}^{n}\right\|_{\psi}^{s}$, where

$$
{ }^{n}\left\|\left(x_{i}\right)_{i=1}^{n}\right\|_{\psi}^{s}:=\left(\sum_{i=1}^{n}\left|x_{i}\right|\right)^{s} \psi_{n}\left(\frac{\left|x_{1}\right|}{\sum_{i=1}^{n}\left|x_{i}\right|}, \ldots, \frac{\left|x_{n}\right|}{\sum_{i=1}^{n}\left|x_{i}\right|}\right) .
$$


To prove the "triangle" inequality we first show that $\|\cdot\|_{\psi}$ is monotone.

Given $\left(p_{i}\right)_{i},\left(a_{i}\right)_{i} \in \Delta_{\infty}$ such that $p_{i} \leq a_{i}$ for each $n \in \mathbb{N}$, there exists some $n \in \mathbb{N}$ with $p_{i}=a_{i}=0$ for all $i>n$. Let us suppose the condition (A) is fulfilled and $0 \leq p_{1} \leq a_{1}$. We denote $a=a_{1}+p_{2}+\ldots p_{n}, p=p_{1}+$ $p_{2}+\ldots p_{n}$ and $\lambda=\frac{a}{p} \geq 1$.

Let $t_{1}=\frac{a_{1}}{\sum a}, t_{i}=\frac{p_{i}}{a}, i=2, \ldots, n$ and $t_{i}^{\prime}=\frac{p_{i}}{\sum p}, i=1, \ldots, n$, then $\frac{1-t_{1}^{\prime}}{1-t_{1}}=\lambda$. Note that $t_{1}^{\prime}=(1-\lambda)+\lambda t_{1}$ and $\lambda\left(1-t_{1}\right)=1-t^{\prime}{ }_{1} \leq 1$. Then $1 \leq \lambda \leq \frac{1}{1-t_{1}}$, and after condition (A), one has

$$
\begin{aligned}
\lambda^{s} \psi_{n}\left(t_{1}, \ldots, \mathrm{t}_{n}\right) & =\lambda^{s} \psi_{n}\left(\frac{a_{1}}{a}, \frac{p_{2}}{a}, \ldots, \frac{p_{n}}{a}\right) \\
& \geq \psi_{n}\left((1-\lambda)+\lambda t_{1}, \lambda t_{2}, \ldots, \lambda t_{n}\right)=\psi_{n}\left(t_{1}^{\prime}, t_{2}^{\prime}, \ldots, t_{n}^{\prime}\right) \\
& =\psi_{n}\left(\frac{p_{1}}{p}, \frac{p_{2}}{p}, \ldots, \frac{p_{n}}{p}\right)
\end{aligned}
$$

i.e.

$$
(a)^{\mathrm{S}} \psi_{n}\left(\frac{a_{1}}{a}, \frac{p_{2}}{a}, \ldots, \frac{p_{n}}{a}\right) \geq(p)^{s} \psi_{n}\left(\frac{p_{1}}{p}, \frac{p_{2}}{p}, \ldots, \frac{p_{n}}{p}\right)
$$

which means

$$
{ }^{n} \mid\left(a_{1}, p_{2}, \ldots, p_{n}\right)\left\|_{\psi}^{s} \geq^{n}\right\|\left(p_{1}, p_{2}, \ldots, p_{n}\right) \|_{\psi}^{s}
$$

Next step is to prove

$$
{ }^{n}\left\|\left(a_{1}, a_{2}, p_{3}, \ldots, p_{n}\right)\right\|_{\psi}^{s} \geq^{n}\left\|\left(a_{1}, p_{2}, p_{3}, \ldots, a_{n}\right)\right\|_{\psi}^{s} .
$$

Since $a_{2} \geq p_{2} \geq 0$, we just act similar to above. In this way, one can get

$$
{ }^{n}\left\|\left(a_{1}, a_{2}, a_{3}, \ldots, a_{n}\right)\right\|_{\psi}^{s} \geq{ }^{n}\left\|\left(a_{1}, p_{2}, p_{3}, \ldots, p_{n}\right)\right\|_{\psi}^{s}
$$

when $a_{i} \geq p_{i}$.

Finally, for triangle inequality one has, by the $s$-convexity of $\psi$,

$$
\begin{aligned}
\| x & +y\|\|_{\psi}^{s}={ }^{n}||\left(x_{1}, \ldots, x_{n}\right)+\left(y_{1}, \ldots, y_{n}\right) \|_{\psi}^{s} \\
= & { }^{n}||\left(x_{1}+y_{1}, \ldots, x_{n}+y_{n}\right)\left\|_{\psi}^{s}={ }^{n}\right\|\left(\left|x_{1}+y_{1}\right|, \ldots,\left|x_{n}+y_{n}\right|\right) \|_{\psi}^{s} \\
\leq & \left.{ }^{n}||\left(\left|x_{1}\right|+\left|y_{1}\right|, \ldots,\left|x_{n}\right|+\left|y_{n}\right|\right)\right|_{\psi} ^{s} \\
= & \left(\sum_{i=1}^{n}\left|x_{i}\right|+\left|y_{i}\right|\right)^{s} \psi_{n}\left(\left(\frac{\left|x_{1}\right|+\left|y_{1}\right|}{\sum_{i=1}^{\infty}\left|x_{i}\right|+\left|y_{i}\right|}, \ldots, \frac{\left|x_{n}\right|+\left|y_{n}\right|}{\sum_{i=1}^{\infty}\left|x_{i}\right|+\left|y_{i}\right|}\right)\right) \\
= & \left(\sum_{i=1}^{n}\left|x_{i}\right|+\left|y_{i}\right|\right)^{s} \psi_{n}\left(\left(\frac{\left|x_{1}\right|}{\sum_{i=1}^{\infty}\left|x_{i}\right|+\left|y_{i}\right|}, \ldots, \frac{\left|x_{n}\right|}{\sum_{i=1}^{\infty}\left|x_{i}\right|+\left|y_{i}\right|}\right)+\right. \\
& \left.+\left(\frac{\left|y_{1}\right|}{\sum_{i=1}^{\infty}\left|x_{i}\right|+\left|y_{i}\right|}, \ldots, \frac{\left|y_{n}\right|}{\sum_{i=1}^{\infty}\left|x_{i}\right|+\left|y_{i}\right|}\right)\right) \\
= & \left(\sum_{i=1}^{n}\left|x_{i}\right|+\left|y_{i}\right|\right)^{s} \psi_{n}\left(\frac{\left|x_{1}\right|+\cdots\left|x_{n}\right|}{\left|x_{1}\right|+\cdots\left|x_{n}\right|}\left(\frac{\left|x_{1}\right|}{\sum_{i=1}^{\infty}\left|x_{i}\right|+\left|y_{i}\right|}, \ldots, \frac{\left|x_{n}\right|}{\sum_{i=1}^{\infty}\left|x_{i}\right|+\left|y_{i}\right|}\right)+\right.
\end{aligned}
$$




$$
\begin{aligned}
& \left.\left.+\frac{\left|y_{1}\right|+\cdots+\left|y_{n}\right|}{\left|y_{1}\right|+\cdots+\left|y_{n}\right|}\left(\frac{\left|y_{1}\right|}{\sum_{i=1}^{\infty}\left|x_{i}\right|+\left|y_{i}\right|}\right), \ldots, \frac{\left|y_{n}\right|}{\sum_{i=1}^{\infty}\left|x_{i}\right|+\left|y_{i}\right|}\right)\right) \\
= & \left(\sum_{i=1}^{n}\left|x_{i}\right|+\left|y_{i}\right|\right)^{s} \psi_{n}\left(\frac{\left|x_{1}\right|+\cdots+\left|x_{n}\right|}{\sum_{i=1}^{\infty}\left|x_{i}\right|+\left|y_{i}\right|}\left(\frac{\left|x_{1}\right|}{\left|x_{1}\right|+\cdots+\left|x_{n}\right|}, \ldots, \frac{\left|x_{n}\right|}{\left|x_{1}\right|+\cdots+\left|x_{n}\right|}\right)+\right. \\
& \left.+\frac{\left|y_{1}\right|+\cdots+\left|y_{n}\right|}{\sum_{i=1}^{\infty}\left|x_{i}\right|+\left|y_{i}\right|}\left(\frac{\left|y_{1}\right|}{\left|y_{1}\right|+\cdots+\left|y_{n}\right|}, \cdots, \frac{\left|y_{n}\right|}{\left|y_{1}\right|+\cdots+\left|y_{n}\right|}\right)\right) \\
\leq & \left(\sum_{i=1}^{n}\left|x_{i}\right|+\left|y_{i}\right|\right)^{s}\left[\left(\frac{\left|x_{1}\right|+\cdots+\left|x_{n}\right|}{\sum_{i=1}^{\infty}\left|x_{i}\right|+\left|y_{i}\right|}\right)^{s} \psi_{n}\left(\frac{\left|x_{1}\right|}{\left|x_{1}\right|+\cdots+\left|x_{n}\right|}, \cdots, \frac{\left|x_{n}\right|}{\left|x_{1}\right|+\cdots+\left|x_{n}\right|}\right)\right)+ \\
& \left.+\left(\frac{\left|y_{1}\right|+\cdots+\left|y_{n}\right|}{\sum_{i=1}^{\infty}\left|x_{i}\right|+\left|y_{i}\right|}\right)^{s} \psi_{n}\left(\frac{\left|y_{1}\right|}{\left|y_{1}\right|+\cdots+\left|y_{n}\right|}, \cdots, \frac{\left|y_{n}\right|}{\left|y_{1}\right|+\cdots+\left|y_{n}\right|}\right)\right] \\
= & \left(\left|x_{1}\right|+\cdots+\left|x_{n}\right|\right)^{s} \psi_{n}\left(\left(\frac{\left|x_{1}\right|}{\left|x_{1}\right|+\cdots+\left|x_{n}\right|}, \cdots, \frac{\left|x_{n}\right|}{\left|x_{1}\right|+\cdots+\left|x_{n}\right|}\right)\right)+ \\
& +\left(\left|y_{1}\right|+\cdots+\left|y_{n}\right|\right)^{s} \psi_{n}\left(\frac{\left|y_{1}\right|}{\left|y_{1}\right|+\cdots+\left|y_{n}\right|}, \cdots, \frac{\left|y_{n}\right|}{\left|y_{1}\right|+\cdots+\left|y_{n}\right|}\right)+ \\
= & \|x\|\left\|_{\psi}^{s}+|| y \mid\right\|_{\psi}^{s} \cdot
\end{aligned}
$$

The proof of Theorem 1 shows that the condition $(A)$ is really closed with monotonicity of the norm.

DEFINITION 7. Given $\psi \in \Psi_{s, \infty}$, the space $\ell_{\psi}$ is defined by

$$
\ell_{\psi}=\left\{\left(x_{n}\right)_{n=1}^{\infty} \in \ell_{\infty}: \lim _{n \rightarrow \infty}\left\|\left(x_{1}, \cdots, x_{n}, 0,0, \ldots\right)\right\|_{\psi}^{s}<\infty\right\},
$$

and $c_{\psi}$ is the closure of $c_{00}$ in $\left(\ell_{\psi},\|\cdot\|_{\psi}\right)$.

The next result may be proved following the proof of Proposition 2.4 in Mitani and Saito (2007).

PROPOSITION 2. The linear space $\ell_{\psi}$ and $c_{\psi}$ are s-Banach spaces with the s-norm $\left\|\left(x_{n}\right)_{n \in \mathbb{N}}\right\|_{\psi}^{s}=$ $\lim _{n \rightarrow \infty}\left\|\left(x_{1}, \cdots, x_{n}, 0,0, \cdots\right)\right\|_{\psi}^{S}$.

EXAMPLE 1. Let $\psi_{p}$ be the p-convex function obtained from the p-norm $\|\cdot\|_{p}$ of $\ell_{p}, 0<p \leq 1$. Then $\psi_{p} \in \Psi_{p, \infty}$ and $\ell_{\psi}=c_{\psi}=\ell_{p}$.

PROPOSITION 3. Let $\psi \in \Psi_{s, \infty}$. Then, for each $\left(x_{n}\right)_{n=1}^{\infty} \in c_{\psi}$ holds

$$
\lim _{n \rightarrow \infty}\left\|\left(0,0, \cdots, 0, x_{n}, x_{n}+1, \cdots\right)\right\|_{\psi}^{s}=0 .
$$

DEFINITION 8. A function $\psi \in \Psi_{s, \infty}$ is called regular if $c_{\psi}=\ell_{\psi}$.

\section{RELATIVE COMPACTNESS AND $\psi$-DIRECT SUMS}

We have considered spaces $\ell_{\psi}$ as spaces of complex numbers labeled in $\mathbb{N}$. An analogous theory may be also developed with labels in $\mathbb{N}_{0}=\mathbb{N} \cup\{0\}$ and even in $\mathbb{Z}$. Therefore, let $\tau: \mathbb{C}^{\mathbb{Z}} \rightarrow \mathbb{C}^{\mathbb{N}}$ be defined by

$$
\tau\left(\left(z_{n}\right)_{n \in \mathbb{Z}}\right)=\left(\hat{z}_{n}\right)_{n \in \mathbb{Z}}
$$


with $\left(\hat{z}_{n}\right)_{2 n+1}=z_{n}$ and $\hat{z}_{2 n}=z_{-n}$, for $n \in \mathbb{N}$. For $\psi \in \Psi_{s, \infty}$ we define

$$
\ell_{\psi}(\mathbb{Z})=\left\{z=\left(z_{n}\right)_{n \in \mathbb{Z}}: \tau(z) \in \ell_{\psi}\right\}
$$

To emphasize which space we are considering, we shall use the notation $\ell_{\psi}(\mathbb{N}), \ell_{\psi}\left(\mathbb{N}_{0}\right)$ and $\ell_{\psi}(\mathbb{Z})$. The next result follows directly from Proposition 2.

PROPOSITION 4. Let $\psi \in \Psi_{s, \infty}$ and $\left(X_{n}\right)_{n \in \mathbb{N}}$ be a sequence of Banach spaces.

Then,

$$
X_{\psi}=\left(\sum_{n \in \mathbb{N}} \oplus X_{n}\right)_{\psi}=\left\{x=\left(x_{n}\right)_{n \in \mathbb{N}} \in \prod_{n \in \mathbb{N}} X_{n}:\left(\left\|x_{n}\right\| X_{n}\right)_{n \in \mathbb{N}} \in \ell_{\psi}\right\}
$$

is an s-Banach space when equipped with the norm $\|x\|=\left\|\left(\left\|x_{n}\right\|\right)_{n \in \mathbb{N}}\right\|_{\psi}$.

In a similar way, let $\left(X_{n}\right)_{n \in \mathbb{Z}}$ be a family of Banach spaces. We define

$$
\left(\sum_{n \in \mathbb{N}} \oplus X_{n}\right)_{\psi}=\left\{\left(x_{n}\right)_{n \in \mathbb{N}}:\left(\left(\left\|x_{n}\right\| X_{n}\right)_{n \in \mathbb{Z}} \in \ell_{\psi}(\mathbb{Z})\right\}\right.
$$

and $\|x\|=\left\|\tau\left(\left\|x_{n}\right\|\right)_{n \in \mathbb{Z}}\right\|_{\ell_{\psi}^{N}}$ for every $x \in\left(\sum_{n \in \mathbb{N}} \oplus X_{n}\right)_{\psi}$.

Examples of $\psi$-direct sums are $\ell_{p}$ direct sums for $\psi=\psi_{p}, 0<p \leq 1$.

Now, the more general situation of $\psi$-direct sum of quasi-Banach spaces is considered.

THEOREM 2. If $\left(X_{n}\right)_{n \in \mathbb{N}}$ is a sequence of quasi-Banach spaces with quasi-Banach constants $C_{n}$, with sup $C_{n}<\infty$, then their $\psi$-direct sum is also a quasi-Banach space.

PROOF. Let $C=\sup C_{n}$. Then,

$$
\begin{aligned}
\|x+y\| & =\left\|\left(\left.\left\|x_{n}+y_{n}\right\|\right|_{X_{n}}\right)\right\|_{\psi} \leq\left\|\left\{\operatorname{Cn}\left(\left\|x_{n}\right\|_{X_{n}}+\left\|y_{n}\right\|_{X_{n}}\right)\right\}\right\| \|_{\psi} \\
& \leq C\left\|\left(\left(\left\|x_{n}\right\|_{X_{n}}+\left\|y_{n}\right\|_{X_{n}}\right)\right)\right\|_{\psi} \leq C C_{\psi}\left(\left\|\left(\left(\left\|x_{n}\right\|_{X_{n}}\left\|_{\psi}+\right\|\left\|y_{n}\right\|_{X_{n}}\right)\right)\right\|_{\psi}\right) \\
& =C C_{\psi}(\|x\|+\|y\|),
\end{aligned}
$$

where $C_{\psi}$ is the quasi-Banach constant of $\ell_{\psi}$.

Using Lemma 1, the completness may be proved as in the Banach case, considered in Proposition 2.4 of Mitani and Saito (2007).

DEFINITION 9. For a bounded set $B$ in $X_{\psi}$, the Hausdorff measure of noncompactness of $B, \chi(B)$, is defined by

$\chi_{X}(B)=\inf \left\{\varepsilon>0\right.$; there exists a finite set $F \subset X$, such that $\left.B \subset F+\varepsilon U_{X}\right\}$, where $U_{X}$ is the closed unit ball of $X_{\psi}$ with center in the origin. 
Now some operators are introduced who will assist in the attainment of the next result.

Let $0<s \leq 1,\left(X_{n}\right)_{n \in \mathbb{N}}$ a sequence of quasi-Banach spaces as in Theorem 2 and $Q_{k}:\left(\sum_{n \in \mathbb{N}} \oplus X_{n}\right)_{\psi} \rightarrow$ $\left(\sum_{i=1}^{k} \oplus X_{n}\right)_{\psi}$, the operator given by $Q_{k}\left(x_{1}, \ldots, x_{n}, \ldots\right)=\left(x_{1}, \ldots, x_{k}\right)$. Then, for $\psi \in \Psi_{s, \infty}$ we define

$$
\left\|Q_{k}(x)\right\|_{\psi_{k}}=\left\|\left(x_{1}, \ldots, x_{k}\right)\right\| \ell_{\psi_{k}}=\left(\sum_{1}^{k}\left\|x_{i}\right\| x_{i}\right)_{\psi_{k}}\left(\frac{\left\|x_{1}\right\|_{X_{1}}}{\sum_{1}^{k}\left\|x_{i}\right\|_{X_{i}}}, \ldots, \frac{\left\|x_{k}\right\|_{X_{1}}}{\sum_{1}^{k}\left\|x_{i}\right\|_{X_{i}}}\right)
$$

if $\left(x_{1}, \ldots, x_{k}\right) \neq(0, \ldots, 0)$, and 0 if $\left(x_{1}, \ldots, x_{k}\right)=(0, \ldots, 0)$, where $\ell_{\psi_{k}}$ is the $s^{-}$space defined on $\mathbb{R}^{k}$.

It is clear that $\left\|Q_{k}(x)\right\|_{\psi_{k}} \leq\left\|Q_{k+1}(x)\right\|_{\psi_{k+1}}$ for every $k=2,3, \ldots$ and $\|x\|_{\psi}=\sup _{k}\left\|Q_{k}(x)\right\|_{\psi_{k}}$.

For a sequence $x=\left(x_{n}\right) \in\left(\sum_{i=1}^{k} \oplus X_{n}\right)_{\psi}$, we also define $P_{k}\left(\left(x_{n}\right)\right)=\left(x_{1}, \ldots, x_{k}, 0,0, \ldots\right)$ and $\pi_{k}\left(\left\{x_{n}\right\}\right)=x_{k}$.

It is clear that $Q_{n}\left(P_{n}(x)\right)=\left(x_{1}, \ldots, x_{n}\right), Q_{n+1}\left(P_{n}(x)\right)=\left(x_{1}, \ldots, x_{n}, 0\right)$ and since $\|\left(\left\|x_{1}\right\|_{X_{1}}, \ldots,\left\|x_{n}\right\|_{X_{n}}\right.$, $0)\left\|_{\psi_{n+1}}=\right\|\left(\left\|x_{1}\right\|_{X_{1}}, \ldots,\left\|x_{n}\right\|_{X_{n}}\right) \|_{\psi_{n}}$, then

$$
\left\|Q_{n}\left(P_{n}(x)\right)\right\|_{\psi_{n}}=\left\|Q_{n+1}\left(P_{n}(x)\right)\right\|_{\psi_{n+1}}=\left\|Q_{n+2}\left(P_{n}(x)\right)\right\|_{\psi_{n+2}}=\ldots
$$

On the other hand since $\left.\| Q_{k}(y)\right)\left\|_{\psi_{k}} \leq\right\| Q_{k+1}(y) \|_{\psi_{k+1}}$, one has

$$
\left\|Q_{n}\left(P_{n}(x)\right)\right\|_{\psi_{n}} \geq\left\|Q_{n-1}\left(P_{n}(x)\right)\right\|_{\psi_{n-1}} \geq \ldots
$$

and

$$
\sup _{k}\left\|Q_{k}\left(P_{n}(x)\right)\right\|_{\psi_{k}}=\left\|Q_{n}\left(P_{n}(x)\right)\right\|_{\psi_{n}},
$$

i.e.

$$
\left.\| P_{n}(x)\right)\left\|x_{\psi}=\right\|\left(\left\|x_{1}\right\|_{X_{1}}, \ldots,\left\|x_{n}\right\|_{X_{n}}\right) \| \ell_{\psi_{n}} .
$$

Hence

$$
\frac{\left\|P_{n}(x)\right\|_{X_{\psi}}}{\|x\|_{X_{\psi}}}=\frac{\left\|\left(\left\|x_{1}\right\|_{X_{1}}, \ldots,\left\|x_{n}\right\|_{X_{n}}\right)\right\| \ell_{\psi_{n}}}{\sup _{k}\left(\left\|x_{1}\right\|_{X_{1}}, \ldots,\left\|x_{n}\right\|_{X_{k}}\right) \| \ell_{\psi_{k}}} \leq 1
$$

i.e. $\left\|P_{n}\right\|_{X_{\psi} \rightarrow X_{\psi}} \leq 1$. If $x=\left(x_{1}, \ldots, x_{n}, 0, \ldots, 0\right)$, then $P_{n}(x)=x$ and thus $\left\|P_{n}\right\|_{X_{\psi} \rightarrow X_{\psi}}=1$.

THEOREM 3. For a bounded subset $B \subset X_{\psi}=\sum_{n \in \mathbb{N}} \oplus X_{n}$ we define

$$
v_{\psi}^{s}(B)=\limsup _{k \rightarrow \infty}\left[\sup _{x \in B}\left\|P_{k}(x)-x\right\|_{X_{\psi}}^{s}+\chi_{X_{\psi}}^{s}\left(P_{k}(B)\right)\right] .
$$

Then if, $\chi_{X_{\psi}}$ is the Hausdorff measure of noncompactness on $X \psi$, one has

$$
\chi_{X_{\psi}}(B) \leq v_{\psi}(B) \leq 2^{\frac{1}{s}} \chi_{X_{\psi}}(B) .
$$

for all bounded subset $B$ in $\chi_{\psi}$.

PROOF. For simplicity, let us denote $\chi_{X_{\psi}}(B)$ by $\chi_{\psi}(B)$. For each bounded subset $B \subset \chi_{\psi}$ and $n \in \mathbb{N}$,

$$
B \subset\left(I d-P_{n}\right) B+P_{n} B
$$


Since $\chi_{\psi}$ is $s$-sub-additive, taking in account the inequality

$$
\chi_{\psi}\left(\left(P_{n}-I d\right) B\right) \leq \sup _{n \in \mathbb{N}}\left\|P_{n} x-x\right\|_{X_{\psi}},
$$

we get

$$
\begin{aligned}
\chi_{\psi}^{s}(B) & \leq \chi_{\psi}^{s}\left(\left(P_{n}-I d\right) B\right)+\chi^{s}\left(P_{n} B\right) \\
& \leq \sup _{x \in B} \|\left(\left(P_{n} x-x\right) \|_{X_{\psi}}^{s}+\chi_{\psi}^{s}\left(P_{n} B\right),\right.
\end{aligned}
$$

for all $n \in \mathbb{N}$. Therefore, $\chi_{\psi}(B) \leq v_{\psi}(B)$.

Conversely, since operators $P_{n}$ are uniformly bounded, let us define $M:=\lim \sup _{n \rightarrow \infty}\left\|P_{n}\right\|$. Then, since $\left\|P_{n}\right\|=1$, it follows $M=1$.

Given a bounded subset $B$ in $X_{\psi}$ and $\varepsilon>0$ arbitrary, let $r=\chi_{\psi}^{s}(B)$ and $r_{\varepsilon}:=r^{\frac{1}{s}}+\varepsilon$. Thus, there is a finite set $B_{0}$ in $X_{\psi}$, such that

$$
B \subset B_{0}+r_{\varepsilon} U_{X_{\psi}} .
$$

And, since $B_{0}$ is finite, there exist $N \in \mathbb{N}$, such that

$$
\left\|\left(P_{n}-I d\right) x_{0}\right\|_{X_{\psi}<\varepsilon^{1 / s}}
$$

for all $n \geq N$ and $x_{0} \in B_{0}$. Now, let $\mathrm{x}$ be an arbitrary element in $B$ and $x_{0} \in B_{0}$ chosen such that $\left\|x-x_{0}\right\|$ $X_{\psi}<r_{\varepsilon}^{1 / s}$. Since

$$
\left\|\left(P_{n}-I d\right) x\right\|_{X_{\psi}}^{s}-\left\|\left(P_{n}-I d\right) x_{0}\right\|_{X_{\psi}}^{s} \leq\left\|\left(P_{n}-I d\right)\left(x-x_{0}\right)\right\|_{X_{\psi}}^{s} \leq r_{\varepsilon},
$$

it holds

$$
\left\|\left(P_{n}-I d\right) x\right\|_{X_{\psi}}^{s} \leq\left\|\left(P_{n}-I d\right) x_{0}\right\|_{X_{\psi}}^{s}+r_{\tilde{\varepsilon}},
$$

and, for all $x \in B$ and $n \geq N$,

$$
\|\left(P_{n} x-x \|_{X \psi}^{s} \leq \varepsilon+r_{\varepsilon}=r^{1 / \mathrm{s}}+2 \varepsilon .\right.
$$

Therefore, taking $\varepsilon \rightarrow 0$, one has

$$
\limsup _{k \rightarrow \infty} \sup _{x \in B}\left\|P_{n} x-x\right\|_{X_{\psi}}^{s} \leq \chi_{\psi}^{s}(B) .
$$

Finally, since $\chi_{\psi}\left(P_{n} B\right) \leq\left\|P_{n}\right\| \chi_{\psi}(B) \leq M \chi_{\psi}(B) \leq \chi_{\psi}(B)$, one has

$$
v^{s}(B) \leq \chi_{\psi}^{s}(B)+\chi_{\psi}^{s}(B)=2 \chi_{\psi}^{s}(B) .
$$

The proof is thus complete.

For a characterization of the compact sets in $\chi_{\psi}$ we need of the following auxiliary result.

LEMMA 2. Let $\pi_{n}: \chi_{\psi} \rightarrow \chi_{n}$ be the natural projection on $\chi_{n}$. Then, for any bounded subset $B \subset \chi_{\psi}$ one has

$$
\chi_{X_{n}}\left(\pi_{n}(B)\right) \leq \chi_{X_{\psi}}(B) .
$$


PROOF. It will be proved that $U_{X_{n}}=\left(\pi_{n}\left(U_{X_{\psi}}\right)\right.$. First we prove $\left\|\pi_{n}(x)\right\|_{X_{n}} \leq\|x\|_{X_{\psi}}$. For any $x=\left(x_{1}, \ldots\right.$, $\left.x_{n}, \ldots\right)$, we put $y=\left(0, \ldots, x_{n}, 0, \ldots, 0, \ldots\right)$. Then

$$
\begin{aligned}
\|x\|_{X_{\psi}} & \geq\|y\|_{X_{\psi}}=\sup _{k}\left\|Q_{k}(y)\right\|_{\psi_{k}}=Q_{n}(y)_{\psi_{n}}=\left\|\left(0, \ldots, 0,\left\|x_{n}\right\|_{X_{n}}\right)\right\| \ell_{\psi_{n}} \\
& =\left.\left\|x_{n}\right\|\right|_{X_{n}}\|(0, \ldots, 0,1)\| \ell_{\psi_{n}}=\left\|x_{n}\right\|\left\|_{X_{n}}=\right\| \pi_{n}(x) \|_{X_{n}} .
\end{aligned}
$$

Hence, if $x \in U_{X_{\psi}}$, then $\pi_{n}(x) \in U_{X_{n}}$.

For a given $z \in U_{X_{n}}$, let $x=(0, \ldots, z, 0, \ldots, 0, \ldots)$, with $z$ in the $n$-th position. Then $\pi_{n}(x)=z$ and $\|x\|_{X_{\psi}}=$ $\|z\|_{X_{n}} \leq 1$. Hence $x \in U_{X_{\psi}}$, which implies that $U_{X_{n}} \in \pi_{n}\left(U_{X_{\psi}}\right)$.

Now, given $\varepsilon>\chi_{\psi}(B)$, there exist balls $B_{1}, \ldots, B_{M} \in X_{\psi}$ which $B_{i}=B\left(x_{i}, \varepsilon\right)$, such that

$$
B \subset \bigcup_{i=1}^{M} \mid B\left(x_{i}, \varepsilon\right)
$$

Thus

$$
\pi_{n}(B) \subset \pi_{n}\left(\bigcup_{i=1}^{M} B\left(x_{i}, \varepsilon\right)\right) \subset \bigcup_{i=1}^{M} \pi_{n}\left(B\left(x_{i}, \varepsilon\right)\right) .
$$

And, since

$$
\pi_{n}\left(B\left(x_{i}, \varepsilon\right)\right)=\pi_{n}\left(x_{i}\right)+\varepsilon \pi_{n}\left(U_{X_{\psi}}\right)=\pi_{n}\left(x_{i}\right)+\varepsilon U_{X_{n}},
$$

for each $i$, there are elements $y_{1}, \ldots, y_{M}$, such that

$$
\left.\pi_{n}(B) \subset \bigcup_{i=1}^{M}\left\{y_{i}+\varepsilon U_{X_{n}}\right)\right\} .
$$

Therefore, $\chi_{X_{n}}\left(\pi_{n}(B)\right) \leq \varepsilon$ and the result follows.

COROLLARY 1. A set $K \subset X_{\psi}$ is relatively compact if, and only if:

(i) $\left\|P_{k} x-x\right\|_{X_{\psi}} \rightarrow 0$ uniformly on $K$, for $k \rightarrow \infty$.

(ii) The set $K(m)=\pi_{m}(K)=\left\{\pi_{m}(x): x \in K\right\}$ is relatively compact in the norm of $X_{m}$ for each $m \in \mathbb{N}$.

PROOF. If $K \subset X_{\psi}$ is relatively compact, then $\chi_{X_{\psi}}(\mathrm{K})=0$ and from Theorem 3.4 we obtain $v_{\psi}(K) \leq$ $\chi_{X_{\psi}}(\mathrm{K})=0$. Hence

$$
0 \leq \limsup _{k \rightarrow \infty}\left[\sup _{x \in K}\left\|P_{k}(x)-x\right\|_{X_{\psi}}\right]=0 .
$$

This means that lim sup is the only one point of condensation of $\sup _{x \in K}\left\|P_{k}(x)-x\right\|_{X_{\psi}}$, hence the lim sup is just a lim. This implies that

$$
\limsup _{k \rightarrow \infty}\left[\sup _{x \in K}\left\|P_{k}(x)-x\right\|_{X_{\psi}}\right]=0,
$$

which means that $\left\|P_{k}(x)-x\right\|_{X_{\psi}} \rightarrow 0$ uniformly on $K$. Hence (i) is fulfilled.

To prove (ii), using Lemma 3.5, one has

$$
\chi_{X_{m}}\left(\pi_{m}(K)\right) \leq \chi_{X_{\psi}}(K),
$$


hence $\chi_{X_{m}}\left(\pi_{m}(K)\right)=0$.

Now, assuming (i) and (ii), the former means that

$$
\limsup _{k \rightarrow \infty}\left[\sup _{x \in B}\left\|P_{k}(x)-x\right\|_{X_{\psi}}\right]=0 .
$$

It follows from (ii) that $\chi_{X_{\psi}}\left(\pi_{k}(K)\right)=0$ and hence $\chi_{X_{\psi}}\left(P_{k}(K)\right)=0$. Then we get $v_{X_{\psi}}(K)=0$ and thus $\chi_{X_{\psi}}(K)$ $=0$, i.e. $K$ is relatively compact.

In particular, if $X$ is a fixed quasi-Banach space and $X_{n}=X$, for each $n \in \mathbb{N}$, we have

$$
X^{p}=p \bigoplus_{n=1}^{\infty} X_{n}=\ell_{p}(X)
$$

Thus, we obtain from Corollary 1 a similar result to that stated by Brooks and Dinculeanu (1979 Thm.1), now for $s$-Banach spaces.

COROLLARY 2. A set $K \subset \ell_{p}(X), 0<p \leq 1$, is relatively compact if, and only if:

(i) $\sum_{m \geq k}\left\|x_{m}\right\|^{p} \rightarrow 0, k \rightarrow \infty$, uniformly for $x \in K$, for $k \rightarrow \infty$.

(ii) for each $m \in \mathbb{N}$, the set $K(m)=\left\{\pi_{m}(x): x \in K\right\}$ is relatively compact in the quasi-norm of $X$.

\section{$s-\psi$-DIRECT SUMS AND INTERPOLATION SPACES}

In Nilsson (1982), the $K$-interpolation space is defined for a pair of quasi-Banach spaces $\left(E_{0}, E_{1}\right)$ and an $\mathbb{Z}$-lattice $A$ (a quasi-Banach space of real valued sequences with $\mathbb{Z}$ as index set and with a monotonicity property: $M$ exists such that $\left|a_{n}\right|_{A} \leq M|| b_{n} \|_{A}$ whenever $\left|a_{n}\right| \leq\left|b_{n}\right|$ for each $n \in \mathbb{Z}$ ).

The $K$-space consists of all $a \in E_{0}+E_{1}$ such that $\left\{K\left(2^{n}, a, E_{0}, E_{1}\right)\right\} \in A$. One more condition has been put on $A$, namely to be $K$-nontrivial, which equivalently may be written as the condition $\left\{\min \left(1,2^{-n}\right)\right\}_{n} \in A$. It will be considered the $K$ interpolation space when $\mathbb{Z}$-lattice is the space

$$
\ell_{\psi}^{\theta}=\left\{\left(z_{n}\right)_{n \in \mathbb{Z}} \in \ell(\mathbb{Z}):\left(2^{-\theta n}\left|z_{n}\right|\right)_{n \in \mathbb{Z}} \in \ell_{\psi}(\mathbb{Z})\right\}
$$

with $\left\|\left(z_{n}\right)_{n \in \mathbb{Z}}\right\|=\left\|\left(2^{-\theta n}\left|z_{n}\right|\right)_{n \in \mathbb{Z}}\right\|_{\psi}$. Due to Lemma 2.4, it is not difficult to see that $\ell_{\psi}^{\theta}$ is $K$-nontrivial, i.e. $(\min (1,2 n))_{n \in \mathbb{Z}} \in \ell_{\psi}^{\theta}$.

Let $\bar{E}=\left(E_{0}, E_{1}\right)$ be a Banach couple, $\theta \in(0,1)$ and $X_{n}=2^{-\theta n} E_{0}+2^{-(\theta-1) n} E_{1}$. The $K$-interpolation space $\left(E_{0}, E_{1}\right)_{\theta, \psi}$ may be defined as the subspace of all constant sequences in $\left(\sum_{n \in \mathbb{Z}} \oplus_{X_{n}}\right)_{\psi}=X_{\psi}$. Since $\|a\|_{X_{n}}=$ $2^{-\theta n} K\left(2^{n}, a, E_{0}, E_{1}\right)$, this is the space of all $a \in E_{0}+E_{1}$, such that $\|a\|_{\theta, \psi} \leq \infty$, where

$$
\|a\|_{\theta, \psi}=\|a\|_{X_{\psi}}=\left\|\left(2^{-\theta n} K\left(2^{n}, a, E_{0}, E_{1}\right)\right)_{n \in \mathbb{Z}}\right\|_{\ell_{\psi}^{\theta}} .
$$

Now, the following characterization may be proved.

THEOREM 4. Let $E_{i}$ be quasi-Banach spaces with quasi-Banach constants $C_{i}, i=0,1$, and let $M$ be the quasi-Banach constant of $c_{\psi}$. Then, the interpolation space $\left(E_{0}, E_{1}\right)_{\theta, \psi}$ is a quasi-Banach space and its quasi-Banach constant does not exceed $M \max \left(C_{0}, C_{1}\right)$. If the norm \|\|$_{\psi}$ is translation invariant, this constant may be estimated by $M 2^{\theta} C_{0}^{1-\theta} C_{1}^{\theta}$. 
PROOF. To prove the first part we will use Lemma 3.11.1 from Bergh and Löfstron (1976). Let $E_{i}$ be quasi-normed with constants $C_{i}, i=0,1$. Then

$$
K\left(t, a+b, E_{0}, E_{1}\right) \leq C_{0}\left(K\left(C_{1} t / C_{0}, a, E_{0}, E_{1}\right)+K\left(C_{1} t / C_{0}, b, E_{0}, E_{1}\right)\right) .
$$

From the inequality $K(t, a) \leq \max (1, t / s) K(s, a)$ we get

$$
\begin{aligned}
C_{0}\left(K\left(C_{1} t / C_{0}, a, E_{0}, E_{1}\right)\right. & \leq C_{0} \max \left(1, C_{1} / C_{0}\right) K\left(t, a, E_{0}, E_{1}\right) \\
& =\max \left(C_{0}, C_{1}\right) K\left(t, a, E_{0}, E_{1}\right),
\end{aligned}
$$

and the same for $b$ in the place of $a$. Thus,

$$
\begin{aligned}
\|a+b\|_{\theta, \psi}= & \left\|\left(2^{-\theta n} K\left(2^{n}, a+\mathrm{b}, E_{0}, E_{1}\right)\right)\right\|_{\ell_{\psi}} \\
\leq & \max \left(C_{0}, C_{1}\right)\left\|2^{-\theta n}\left(K\left(2^{n}, a, E_{0}, E_{1}\right)+K\left(2^{n}, b, E_{0}, E_{1}\right)\right)\right\|_{\ell_{\psi}} \\
\leq & M \max \left(C_{0}, C_{1}\right)\left(\left\|\left(2^{-\theta n} K\left(2^{n}, a, E_{0}, E_{1}\right)\right)\right\|_{\ell_{\psi}}\right. \\
& \left.+\left\|\left(2^{-\theta n} K\left(2^{n}, b, E_{0}, E_{1}\right)\right)\right\| \|_{\psi}\right) \\
= & M \max \left(C_{0}, C_{1}\right)\left(\|a\|_{\theta, \psi}+\|b\|_{\theta, \psi}\right) .
\end{aligned}
$$

In the case of the second part of the theorem, from (3)

$$
\begin{aligned}
\|a+b\|_{\theta, \psi}= & \left\|\left(2^{-\theta n} K\left(2^{n}, a+\mathrm{b}, E_{0}, E_{1}\right)\right)\right\|_{\ell_{\psi}} \\
\leq & \left\|\left(2^{-\theta n} C_{0}\left(K\left(2^{n} C_{1} / C_{0}, a, E_{0}, E_{1}\right)+K\left(2^{n} C_{1} / C_{0}, b, E_{0}, E_{1}\right)\right)\right)\right\|_{\ell_{\psi}} \\
\leq & M C_{0}\left\|2^{-n \theta} K\left(2^{n} C_{1} / C_{0}, a, E_{0}, E_{1}\right)\right\|_{\ell_{\psi}} \\
& \left.+\left\|\left(2^{-n \theta} K\left(2^{n} C_{1} / C_{0}, b, E_{0}, E_{1}\right)\right)\right\|_{\ell_{\psi}}\right) .
\end{aligned}
$$

Now, choose $m \in \mathbb{Z}$, such that $2^{m} \leq C_{1} / C_{0} \leq 2^{m+1}$. Then, since $\|\cdot\|_{\ell_{\psi}}$ is translation invariant, we have

$$
\begin{aligned}
\left\|2^{-n \theta} K\left(2^{n} C_{1} / C_{0}^{\prime}, a, E_{0}, E_{1}\right)\right\|_{\ell_{\psi}} & \leq\left\|2^{-n \theta} K\left(2^{n+m+1}, a, E_{0}, E_{1}\right)\right\|_{\ell_{\psi}} \\
& =\left\|2^{-(n+m+1) \theta} 2^{(m+1) \theta} K\left(2^{n+m+1}, a, E_{0}, E_{1}\right)\right\|_{\ell_{\psi}} \\
& =2^{(m+1) \theta}\|a\|_{\theta, \psi} \\
& \left(\frac{C_{1}}{C_{0}}\right)^{\theta} 2^{\theta}\|a\|_{\theta, \psi} .
\end{aligned}
$$

The same is obtained to $\left\|2^{-n \theta} K\left(2^{n} C_{1} / C_{0}, b, E_{0}, E_{1}\right)\right\|_{\ell_{\psi}}$. Therefore,

$$
\|a+b\|_{\theta, \psi} \leq M 2^{\theta} C_{0}^{1-\theta}\left(\|a\|_{\theta, \psi}+\|b\|_{\theta, \psi}\right) \text {. }
$$

Now, we may get a result about relative compactness and $s-\psi$-interpolation spaces using a modification of results from the previous section.

For a sequence $x=\left(x_{n}\right) n \in \mathbb{Z}$, let us consider the $\tau$ operator from Section 3,

$$
\tau(x)=\tau\left(\left(x_{n}\right)\right)=\left(x_{0}, x_{-1}, x_{1}, x_{-2}, x_{2}, \ldots, x_{-n}, x_{n}, \ldots\right),
$$

and

$$
\tau(x)-P_{2 k+1} \tau(x)=\left(0, \ldots 0, x_{-(n+1)}, x_{(n+1)}, x_{-(n+2)}, x_{(n+2)}, \ldots\right) .
$$

Then, it is appropriate to consider a modification of the measure of noncompactness $v$, namely

$$
\tilde{v}_{\psi}(B)=\limsup _{k \rightarrow \infty}\left[\sup _{x \in B}\left\|P_{2 k+1} \tau(x)-\tau(x)\right\|_{X_{\psi}}+\chi_{X_{\psi}}\left(P_{2 k+1}(B)\right)\right] .
$$


The results for this measure are analogous to those given in Theorem 3 for the measure $v_{\psi}(B)$. Thus the following theorem for the compactness of bounded sets in the $\psi$-interpolation spaces may be obtained:

THEOREM 5. Given quasi-Banach spaces $E_{0}$ and $E_{1}$, a bounded set $K$ is relatively compact in $\left(E_{0}, E_{1}\right)_{\theta, \psi}$, for $0<\theta<1$ and $\psi$ regular if, and only if,

(i) $\lim _{n \rightarrow \infty}\left\|\left\{c(k, n) K\left(2^{k}, a, E_{0}, E_{1}\right)\right\}\right\|_{\ell_{\psi}}=0$ uniformly in $x \in K$, where $c(k, n)$ equals $2^{-\theta k}$ if $|k| \geq \mathrm{n}$ and 0 , if $k=-n,-n+1, \ldots, n-1, n$.

(ii) The set $K$ is relatively compact in $E_{0}+E_{1}$.

Here we use the fact that if $K$ is relatively compact in $E_{0}+E_{1}$ then, it is also relatively compact in $X_{n}=2^{-\theta n} E_{0}+2^{-(\theta-1) n} E_{1}$.

When $\psi=\psi_{p}$, is obtained for the quasi-Banach case the Theorem 3.2 from Fernandez and Silva (2006), which for the Banach case is a result originally given in Peetre (1968) and also proved in Fernandez-Cabrera (2002). Similar results for general real interpolation methods appear in Cobos, et al. (2005).

\section{ACKNOWLEDGMENTS}

Research of L. Nikolova was partially supported by Sofia University SRF under contract \#133/2012. The authors would like to thank to the anonymous referees for helpful corrections and suggestions.

\section{RESUMO}

Dado $0<s \leq 1$ e uma função s-convexa $\psi$, os espaços de sequencias $s-\psi$ são introduzidos. Vários espaços quaseBanach de sequencias são assim caracterizados como um caso particular dos espaços $s-\psi$. Para esses espaços novas medidas de não compacidade são também definidas, relacionadas a medida de não compacidade de Hausdorff. Como uma aplicação, conjuntos compactos nos espa,cos de interpolação $s-\psi$, de um par quase-Banach são estudados.

Palavras-chave: espaços quase-Banach, funções quase-convexas, medidas de não compacidade, teoria de interpolação.

\section{REFERENCES}

ALBIAC F AND KALTON NJ. 2009. Lipschitz structure of quasi-Banach spaces. Israel J Math 170: 317-335.

AlBiAC F AND LERÁNOZ C. 2010a. Drops in quasi-Banach spaces. J Geom Anal 20: 525-537.

AlBIAC F AND LERÁNOZ C. 2010b. Uniqueness of unconditional basis in quasi-Banach spaces which are not sufficiently Euclidean. Positivity 14: 579-584.

AOKI T. 1942. Locally bounded linear topological spaces. Proc Imp Acad Tokyo 18: 588-594.

BANÁS J AND GOEBEL K. 1980. Measures of Noncompactness in Banach Spaces, Marcel Dekker, 97 p.

BERGH J AND COBOS F. 2000. A maximal description for the real interpolation method in the quasi-Banach case. Math Scand 87: 22-26.

BERGH J AND LÖFSTRON J. 1976. Interpolation Spaces: An Introduction. Springer-Verlag, 207 p.

BownIK M. 2005. Boundedness of operators on Hardy spaces via atomic decompositions. Proc Amer Math Soc 133(12): $3535-3542$.

BownIK M, Li B, YANG D AND ZHOU Y. 2010. Weighted anisotropic product Hardy spaces and boundedness of sublinear operators. Math Nachr 283(3): 392-442.

BROOKS JK AND DiNCULEANU N. 1979. Conditional expectation and weak and strong compactness in spaces of Bochner integrable functions. J Mult Anal 9: 420-427.

CALDERÓN AP. 1964. Intermediate spaces and interpolation, the complex method. Studia Math 24: 113-190.

Cobos F, FernándeZ-CABrera LM AND MartíneZ A. 2005. Compact operators between K- and J-spaces. Studia Math 166: 199-220.

Cobos F, Fernández-CABrera LM, MANZANo A AND MARTínez A. 2007. Logarithmic interpolation spaces between quasiBanach spaces. Zeit Fur Anal Anwendungen 26(1): 65-86.

CoBOs F AND PERSSON LE. 1998. Real interpolation of compact operators between quasi-Banach spaces. Math Scand 82: $138-160$. 
DUNFORD N AND SCHWARTZ J. 1967. Linear Operators - Part I. Interscience Pub. Inc., New York, 858 p.

FERNANDEZ DL AND SILVA EB. 2006. Hausdorff measures of noncompactness and interpolation spaces. Serdica Math J 32(2-3): 179-184.

FERNANDEZ DL AND SILVA EB. 2010. Generalized measures of noncompactness of sets and operators in Banach spaces. Acta Math Hungarica 129: 227-244.

FERNÁNDEZ-CABRERA LM. 2002. Compact operators between real interpolation spaces. Math Ineq Appl 5: 283-289.

Gomez LAP AND Silva EB. 2011. Vector-valued singular integral operators on product Hardy space $H^{p}$. Indian J Math 53(2): 339-369.

GHorbani Z AND ModArRes SMS. 2007. Minimal and maximal description for the real interpolation methods in the case of quasi-Banach n-tuples. Int. J Math Analysis 1(14): 689-696.

GRAFAKOS L AND MASTYLO M. 2006. Interpolation of bilinear operators between quasi-Banach spaces. Positivity 10: 409-429.

HOLMSTED T. 1970. Interpolation of quasi-normed spaces. Math Scand 26: 177-199.

HudziK H AND MaLigranda L. 1994. Some remarks on s-convex functions. Aequationes Math 48: 100-111.

Kalton NJ, Peck NT and Rogers JW. 1985. An F-Space Sampler. London Math. Lecture Notes, vol. 89. Cambridge Univ. Press, Cambridge.

KRÉE P. 1967. Interpolation d'espaces qui ne sont ni normés ni complets. Applications. Ann Inst Fourier 17: 137-174.

Lions JL and PeEtre J. 1964. Sur une classe d'espaces d'interpolation. Pub Math de l'I H E S 19: 5-68.

Mastylo M AND MLECZKo P. 2010. Solid hulls of quasi-Banach spaces of analytic functions and interpolation. Nonl Anal 73: 84-98.

MitANI K AND SAITO KS. 2007. On genezalized $\ell_{p}$-spaces. Hiroshima Math J 37: 1-12.

MOLINA JAL. 2009. Existence of complemented subspaces isomorphic to $\ell^{q}$ in quasi Banach interpolation spaces. Rocky Mt J Math 39(3): 899-926.

NiKOlOVA L AND ZaCHARIAdeS T. 2009. On $\psi$ interpolation spaces. Math Ineq Appl 12(4): 827-838.

NILSSON P. 1982. Reiteration theorems for real interpolation and approximation spaces. Ann Math Pura Appl 132: 291-330.

Peetre J. 1968. A theory of interpolation of normed spaces. Lecture notes, Brasilia 1963 [Notas Mat. 39].

PeETRE J. 1970. A new approach in interpolations spaces. Studia Math 34: 23-42.

PHILLIPS RS. 1940. On linear transformations. Trans Amer Math Soc 48: 516-541.

RoLEWICZ S. 1957. On a certain class of linear metric spaces. Bull Acad Pol Sci Cl III(5): 471-473.

RolewiCZ S. 1985. Metric Linear Spaces. In: Mathematics and Its Applications, $2^{\text {nd }}$ ed., East European Series, vol. 20. Reidel, Dordrecht.

SAGHER Y. 1972. Interpolation of r-Banach spaces. Stud Math 41: 45-70.

ZACHARIADES T. 2011. On $\ell_{\psi}$ spaces and infinite $\psi$-direct sums of Banach spaces. Rocky Mt J Math 41(3): 971-997. 